\title{
Aumann Integral on Time Scales
}

\section{Iguer Luis Domini Dos Santos}

Department of Mathematics, Sao Paulo State University, Ilha Solteira, São Paulo, Brazil

Email: iguerluis@mat.feis.unesp.br

How to cite this paper: Dos Santos, I.L.D. (2018) Aumann Integral on Time Scales. Open Access Library Journal, 5: e4254. https://doi.org/10.4236/oalib.1104254

Received: December 13, 2017

Accepted: January 13, 2018

Published: January 16, 2018

Copyright $\odot 2018$ by author and Open Access Library Inc.

This work is licensed under the Creative Commons Attribution International License (CC BY 4.0).

http://creativecommons.org/licenses/by/4.0/

\begin{abstract}
In this paper, we consider by the first time the Aumann integral on time scales. Hence, we introduce the Aumann $\Delta$-integral on time scales. We also have established properties for the Aumann $\Delta$-integral on time scales. In particular, we obtain a formula that relates the Aumann $\Delta$-integral on time scales and the Aumann integral.
\end{abstract}

\section{Subject Areas}

Mathematical Analysis

\section{Keywords}

Aumann Integral, Time Scales, Lebesgue $\Delta$-Integral on Time Scales

\section{Introduction}

Integrals on time scales were considered, for example, by Liu and Zhao [1], Mozyrska et al. [2] and by Peterson and Thompson [3]. Liu and Zhao [1] studied the McShane integral on time scales. On the other hand, Mozyrska et al. [2] studied the Riemann-Stieltjes integral on time scales. In turn, Peterson and Thompson [3] studied the Henstock-Kurzweil integral on time scales. Here we establish an extension of the Aumann integral. Thus, using the Lebesgue $\Delta$-integral on time scales, see for example Guseinov [4], we define the Aumann $\Delta$-integral on time scales. To the best of our knowledge, the Aumann integral on time scales has not yet been considered in the literature. We get some basic properties for the Aumann $\Delta$-integral on time scales in consonance with the basic properties of the Aumann integral considered by Aumann [5]. Furthermore, we established a formula that relates the Aumann $\Delta$-integral on time scales and the Aumann integral, in analogy to the formula obtained by Cabada and Vivero [6] that relates the Lebesgue $\Delta$-integral on time scales and the Lebesgue integral. 


\section{Preliminaries}

In this section we consider concepts and results necessary for the study of the Aumann $\Delta$-integral on time scales.

\subsection{Time Scales}

A time scale is a nonempty closed subset $\mathbb{T} \subset \mathbb{R}$ of the real numbers. Here we use an arbitrary bounded time scale $\mathbb{T}$ where $a=\min \mathbb{T}$ and $b=\max \mathbb{T}$ are such that $a<b$.

Define the forward jump operator $\sigma: \mathbb{T} \rightarrow \mathbb{T}$ by

$$
\sigma(t)=\inf \{s \in \mathbb{T}: s>t\} .
$$

Here we assume that $\inf \varnothing=\sup \mathbb{T}$.

Lemma 1 [6] There exist $I \subset \mathbb{N}$ and $\left\{t_{i}\right\}_{i \in I} \subset \mathbb{T}$ such that

$$
R S:=\{t \in \mathbb{T}: t<\sigma(t)\}=\left\{t_{i}\right\}_{i \in I},
$$

where RS stands for right scattered points of the time scale $\mathbb{T}$.

\subsection{Lebesgue Integration on Time Scales}

The definition of $\Delta$-mensurable sets of $\mathbb{T}$, was considered, for example, by Guseinov [4].

We denote the family of $\Delta$-mensurable sets of $\mathbb{T}$ by $\Delta$. We remember that $\Delta$ is a $\sigma$-algebra of subsets of the time scale $\mathbb{T}$.

It is said that a function $f: \mathbb{T} \rightarrow \overline{\mathbb{R}}$ is $\Delta$-measurable if for each $r \in \mathbb{R}$ the set $\{t \in \mathbb{T}: f(t)<r\}$ is $\Delta$-measurable. The vector valued function $f: \mathbb{T} \rightarrow \mathbb{R}^{n}$ is $\Delta$-measurable if each component $f_{i}: \mathbb{T} \rightarrow \mathbb{R}$ is $\Delta$-measurable.

Consider a function $f: \mathbb{T} \rightarrow \overline{\mathbb{R}}$ and a set $E \in \Delta$. We indicate by

$$
\int_{E} f(s) \Delta s
$$

the Lebesgue $\Delta$-integral of $f$ over $E$. If $f: \mathbb{T} \rightarrow \mathbb{R}^{n}$ is a $\Delta$-measurable function and $E \in \Delta, f$ is integrable over $E$ if each component $f_{i}: \mathbb{T} \rightarrow \mathbb{R}$ is integrable over $E$. In this case

$$
\int_{E} f(s) \Delta s=\left(\int_{E} f_{1}(s) \Delta s, \cdots, \int_{E} f_{n}(s) \Delta s\right) .
$$

We denote by $L_{1}\left(E, \mathbb{R}^{n}\right)$ the set of functions $f: \mathbb{T} \rightarrow \mathbb{R}^{n} \Delta$-integrable over E.

Cabada and Vivero [6] and Santos and Silva [7] consider a more complete approach to Lebesgue integration theory on time scales.

Given a function $f: \mathbb{T} \rightarrow \mathbb{R}^{n}$, define $\tilde{f}:[a, b] \rightarrow \mathbb{R}^{n}$ as

$$
\tilde{f}(t)=\left\{\begin{array}{l}
f(t), t \in \mathbb{T} \\
f\left(t_{i}\right), t \in\left(t_{i}, \sigma\left(t_{i}\right)\right) \text { for some } i \in I,
\end{array}\right.
$$

where $I \subset \mathbb{N}$ and $\left\{t_{i}\right\}_{i \in I}=R S$.

If $E \subset \mathbb{T}$, define 


$$
\tilde{E}=E \bigcup \bigcup_{i \in I_{E}}\left(t_{i}, \sigma\left(t_{i}\right)\right)
$$

where

$$
I_{E}:=\left\{i \in I: t_{i} \in E \cap R S\right\} .
$$

It follows from Cabada and Vivero [6] the next two results.

Proposition 1 Take a function $f: \mathbb{T} \rightarrow \mathbb{R}^{n}$. Then $f$ is $\Delta$-measurable if and only if $\tilde{f}$ is Lebesgue measurable.

Theorem 1 Let $E \in \Delta$ be such that $b \notin E$. Then $f \in L_{1}\left(E, \mathbb{R}^{n}\right)$ if and only if $\tilde{f} \in L_{1}\left(\tilde{E}, \mathbb{R}^{n}\right)$. In this case

$$
\int_{E} f(s) \Delta s=\int_{\tilde{E}} \tilde{f}(s) \mathrm{d} s
$$

\subsection{Measurable Multifunctions}

Let $(\Omega, \mathcal{F})$ be a measurable space. A multifunction is a set-valued function $\Gamma: \Omega \rightsquigarrow \mathbb{R}^{n}$ that takes points $x \in \Omega$ into subsets $\Gamma(x)$ of $\mathbb{R}^{n}$. We say that the multifunction $\Gamma: \Omega \rightsquigarrow \mathbb{R}^{n}$ is $\mathcal{F}$-measurable if the set

$$
\Gamma^{-1}(V)=\{x \in \Omega: \Gamma(x) \cap V \neq \varnothing\}
$$

is $\mathcal{F}$-measurable for all compact sets $V \subset \mathbb{R}^{n}$.

A function $\gamma: \Omega \rightarrow \mathbb{R}^{n}$ is a selection of the multifunction $\Gamma$ if $\gamma(x) \in \Gamma(x)$ for each $x \in \Omega$.

A multifunction $\Gamma$ is said to be closed, compact, convex or nonempty when $\Gamma(x)$ satisfies the required property, for each point $x \in \Omega$.

We will use the following result due to Castaing and Valadier [8].

Theorem 2 Let $(\Omega, \mathcal{F})$ be a measurable space and $\Gamma: \Omega \rightsquigarrow \mathbb{R}^{m}$ a nonempty closed multifunction. If $\Gamma$ is $\mathcal{F}$-measurable then $\Gamma$ admits a measurable selection.

\section{Aumann $\Delta$-Integral on Time Scales}

If $A \subset \mathbb{R}$, we denote the set $A \cap \mathbb{T}$ by $A_{\mathbb{T}}$.

Consider a nonempty multifunction $F: \mathbb{T} \rightsquigarrow \mathbb{R}^{n}$. Let $\mathcal{F}$ be the set of all functions $\quad f: \mathbb{T} \rightarrow \mathbb{R}^{n}$ such that $f$ is $\Delta$-integrable over $[a, b)_{\mathbb{T}}$ and $f(t) \in F(t)$ for all $t \in \mathbb{T}$. We define the Aumann $\Delta$-integral of $F$ over $[a, b)_{\mathbb{T}}$ by

$$
\int_{[a, b)_{\mathbb{T}}} F(s) \Delta s=\left\{\int_{[a, b)_{\mathbb{T}}} f(s) \Delta s: f \in \mathcal{F}\right\} .
$$

We note that the Aumann $\Delta$-integral of $F$ over $[a, b)_{\mathbb{T}}$ coincides with the usual Aumann integral when $\mathbb{T}=[a, b]$. Hence the Aumann $\Delta$-integral on time scales is a generalization of the usual Aumann integral.

From definition, if $\mathbb{T}=\{0,1\}$ and $F: \mathbb{T} \rightsquigarrow \mathbb{R}$ is given by $F(t)=\{0,1\}$ for each $t \in \mathbb{T}$, then $\int_{[0,1)_{\mathbb{T}}} F(s) \Delta s=\{0,1\}$. On the other hand, if $\mathbb{T}=[0,1]$ and $F: \mathbb{T} \rightsquigarrow \mathbb{R}$ is defined by $F(t)=\{0,1\}$ for every $t \in \mathbb{T}$, then 


$$
\int_{[0,1)_{\mathbb{T}}} F(s) \Delta s=[0,1] .
$$

Below we establish properties for the Aumann $\Delta$-integral on time scales.

Theorem 3 If $F: \mathbb{T} \rightsquigarrow \mathbb{R}^{n}$ is a convex nonempty multifunction, then $\int_{[a, b)_{T}} F(s) \Delta s$ is convex.

Proof. Let $f_{1}, f_{2} \in \mathcal{F}$. If $\alpha \in[0,1]$ it follows that $\alpha f_{1}+(1-\alpha) f_{2} \in \mathcal{F}$. Hence,

$$
\begin{aligned}
& \alpha \int_{[a, b)_{\mathbb{T}}} f_{1}(s) \Delta s+(1-\alpha) \int_{[a, b)_{\mathbb{T}}} f_{2}(s) \Delta s \\
& =\int_{[a, b)_{\mathbb{T}}}\left(\alpha f_{1}+(1-\alpha) f_{2}\right)(s) \Delta s \in \int_{[a, b)_{\mathbb{T}}} F(s) \Delta s
\end{aligned}
$$

and thus $\int_{[a, b)_{\mathbb{T}}} F(s) \Delta s$ is convex.

We say that the multifunction $F: \mathbb{T} \rightsquigarrow \mathbb{R}^{n}$ is $\Delta$-integrably bounded if there is a function $c: \mathbb{T} \rightarrow[0,+\infty) \Delta$-integrable over $[a, b)_{\mathbb{T}}$ such that $\|y\| \leq c(t)$ for all $y$ and $t$ such that $y \in F(t)$.

Theorem 4 Let $F: \mathbb{T} \rightsquigarrow \mathbb{R}^{n}$ be a nonempty closed, $\Delta$-integrably bounded and $\Delta$-measurable multifunction. Then $\int_{[a, b)_{\mathbb{T}}} F(s) \Delta s$ is nonempty.

Proof. From Theorem 2 the multifunction $F$ admits a $\Delta$-measurable selection $f$. Since $F$ is $\Delta$-integrably bounded, it follows that $f$ is $\Delta$-integrable over $[a, b)_{\mathbb{T}}$. Thus, $f \in \mathcal{F}$ and then $\int_{[a, b)_{\mathbb{T}}} F(s) \Delta s$ is nonempty.

Given a multifunction $F: \mathbb{T} \rightsquigarrow \mathbb{R}^{n}$, we define the multifunction $\tilde{F}:[a, b] \rightsquigarrow \mathbb{R}^{n}$ by

$$
\tilde{F}(t)=\left\{\begin{array}{l}
F(t), t \in \mathbb{R} \\
F\left(t_{i}\right), t \in\left(t_{i}, \sigma\left(t_{i}\right)\right) \text { for some } i \in I .
\end{array}\right.
$$

Theorem 5 Let $F: \mathbb{T} \rightsquigarrow \mathbb{R}$ be a nonempty compact and convex multifunction. Then

$$
\int_{[a, b)_{\mathbb{T}}} F(s) \Delta s=\int_{[a, b]} \tilde{F}(s) \mathrm{d} s .
$$

Proof. Let $f: \mathbb{T} \rightarrow \mathbb{R}$ be a selection of $F$. Suppose that $f$ is $\Delta$-integrable over $[a, b)_{\mathbb{T}}$. Hence the function $\tilde{f}:[a, b] \rightarrow \mathbb{R}$ is a selection of $\tilde{F}$. Furthermore, it follows from Theorem 1 that

$$
\int_{[a, b)_{\mathbb{T}}} f(s) \Delta s=\int_{[a, b)} \tilde{f}(s) \mathrm{d} s=\int_{[a, b]} \tilde{f}(s) \mathrm{d} s
$$

and therefore

$$
\int_{[a, b)_{\mathbb{T}}} F(s) \Delta s \subset \int_{[a, b]} \tilde{F}(s) \mathrm{d} s .
$$

Consider a selection $g:[a, b] \rightarrow \mathbb{R}$ of $\tilde{F}$. Suppose that $g$ is Lebesgue integrable over $[a, b]$.

Let $A=\bigcup_{i \in I}\left(t_{i}, \sigma\left(t_{i}\right)\right)$. We have

$$
\begin{aligned}
\int_{[a, b]} g(s) \mathrm{d} s & =\int_{[a, b)} g(s) \mathrm{d} s=\int_{A \cup([a, b) \backslash A)} g(s) \mathrm{d} s \\
& =\int_{A} g(s) \mathrm{d} s+\int_{[a, b) \backslash A} g(s) \mathrm{d} s
\end{aligned}
$$




$$
=\sum_{i \in I} \int_{\left(t_{i}, \sigma\left(t_{i}\right)\right)} g(s) \mathrm{d} s+\int_{[a, b) \backslash A} g(s) \mathrm{d} s .
$$

Since $F$ is a compact and convex multifunction, for each $i \in I$ there exists $\beta_{i} \in F\left(t_{i}\right)$ such that

$$
\int_{\left(t_{i}, \sigma\left(t_{i}\right)\right)} g(s) \mathrm{d} s=\beta_{i}\left(\sigma\left(t_{i}\right)-t_{i}\right) .
$$

Define the function $h: \mathbb{T} \rightarrow \mathbb{R}$ as

$$
h(t)= \begin{cases}\beta_{i}, & \text { if } t=t_{i} \text { for some } i \in I \\ g(t), & \text { if } \sigma(t)=t .\end{cases}
$$

Then

$$
\int_{[a, b)} g(s) \mathrm{d} s=\int_{[a, b)} \tilde{h}(s) \mathrm{d} s=\int_{[a, b)_{\mathbb{T}}} h(s) \Delta s
$$

and thus

$$
\int_{[a, b]} \tilde{F}(s) \mathrm{d} s \subset \int_{[a, b)_{\mathbb{T}}} F(s) \Delta s .
$$

Hence the proof is complete.

Theorem 6 Let $F: \mathbb{T} \rightsquigarrow \mathbb{R}$ be a nonempty compact, convex and $\Delta$-integrably bounded multifunction. Then $\int_{[a, b)_{\mathbb{T}}} F(s) \Delta s$ is a compact set.

Proof. We know by Aumann [5] that the set

$$
\int_{[a, b]} \tilde{F}(s) \mathrm{d} s
$$

is compact. From Theorem 5 we may conclude that the set

$$
\int_{[a, b)_{\mathbb{T}}} F(s) \Delta s
$$

is compact.

\section{Conclusion}

By introducing the Aumann $\Delta$-integral on time scales, the paper contributes to the theory of time scales, more specifically, for the integration on time scales. The Aumann integral on time scales is added to other extensions of integrals for the theory of time scales, namely, the McShane integral on time scales, the Riemann-Stieltjes integral on time scales and the Henstock-Kurzweil integral on time scales, among others. The paper also established properties for the Aumann $\Delta$-integral on time scales. Moreover, a formula is also established that relates the Aumann $\Delta$-integral on time scales and the Aumann integral. However, such a formula is restricted to multifunctions $F: \mathbb{T} \rightsquigarrow \mathbb{R}$. Thus, future work might consider possibilities under which this formula remains valid for multifunctions $F: \mathbb{T} \rightsquigarrow \mathbb{R}^{n}$.

\section{References}

[1] Liu, D. and Zhao, D. (2012) On the McShane Integral on Time Scales. Chinese Quarterly Journal of Mathematics, 27, 556-561.

[2] Mozyrska, D., Pawłuszewicz, E. and Torres, D.F.M. (2010) The Riemann-Stieltjes 
Integral on Time Scales. The Australian Journal of Mathematical Analysis and Applications, 7, 1-14.

[3] Peterson, A. and Thompson, B. (2006) Henstock-Kurzweil Delta and Nabla Integrals. Journal of Mathematical Analysis and Applications, 323, 162-178. https://doi.org/10.1016/j.jmaa.2005.10.025

[4] Guseinov, G.S. (2003) Integration on Time Scales. Journal of Mathematical Analysis and Applications, 285, 107-127. https://doi.org/10.1016/S0022-247X(03)00361-5

[5] Aumann, R.J. (1965) Integrals of Set-Valued Functions. Journal of Mathematical Analysis and Applications, 12, 1-12. https://doi.org/10.1016/0022-247X(65)90049-1

[6] Cabada, A. and Vivero, D.R. (2006) Expression of the Lebesgue $\Delta$-Integral on Time Scales as a Usual Lebesgue Integral: Application to the Calculus of $\Delta$-Antiderivatives. Mathematical and Computer Modelling, 43, 194-207. https://doi.org/10.1016/j.mcm.2005.09.028

[7] Santos, I.L.D. and Silva, G.N. (2013) Absolute Continuity and Existence of Solutions to Dynamic Inclusions in Time Scales. Mathematische Annalen, 356, 373-399. https://doi.org/10.1007/s00208-012-0851-8

[8] Castaing, C. and Valadier, M. (1977) Convex Analysis and Measurable Multifunctions. Springer Lecture Notes in Mathematics, Vol. 580.

https://doi.org/10.1007/BFb0087685 